ARTÍCULO ORIGINAL

\title{
Dengue hemorrágico en niños: diez años de experiencia clínica
}

\author{
Ángela Méndez, Gerardo González \\ Departamento de Pediatría, Universidad Industrial de Santander, \\ Hospital Universitario Ramón González Valencia, Bucaramanga, Colombia.
}

En Bucaramanga, Colombia, el dengue hemorrágico se presenta en forma endemoepidémica desde 1992. Con el objetivo de mostrar nuestras propias observaciones sobre las características clínicas, de laboratorio y evolución de la enfermedad realizamos este estudio durante un período de 10 años (febrero de 1992 a febrero de 2002), en niños menores de 13 años hospitalizados en el hospital universitario. Se incluyeron 763 pacientes, de los cuales 617 fueron clasificados como dengue hemorrágico de acuerdo con los criterios de la OMS $(9,2 \%$, grado I; $61,5 \%$, grado II; $21,7 \%$, grado III, y 7,5\%, grado IV). No se pudieron clasificar 146 pacientes. La mayor incidencia se presentó en 1997, 1998 y 2001. Del total, 74,9\% procedía del área metropolitana de Bucaramanga; 48\% eran del género masculino; 0,3\% eran recién nacidos; lactantes, 11,8\%; preescolares, 23,1\%, y escolares, 64,9\%. Las principales manifestaciones clínicas fueron: fiebre, 100\%; manifestaciones hemorrágicas, 100\%; vómito, $60 \%$; dolor abdominal, 57\%; cefalea, 50\%; dolor osteomuscular, 40,8\%; hepatomegalia, 33\%, y exantemas, $29,4 \%$. Entre las manifestaciones hemorrágicas, $56 \%$ correspondió a petequias; $35 \%$ a prueba de torniquete positiva; 34\% a hemorragias digestivas, y $32 \%$ a epistaxis. Presentaron derrames serosos 17,7\%. Entre los signos de alarma para choque se destacó el dolor abdominal intenso. En $52 \%$ de los pacientes se encontró leucopenia y linfocitos atípicos en $37,3 \%$. Entre otras manifestaciones inusuales se presentaron hepatitis, encefalopatía, colecistitis acalculosa, insuficiencia renal aguda, síndrome hemofagocítico, infecciones sobreagregadas y enfermedades concomitantes. La evolución fue favorable en la mayoría de los pacientes. Fallecieron 12 pacientes (1,5\%).

Palabras clave: dengue hemorrágico, niños.

Dengue haemorrhagic fever in children: ten years of clinical experience

In Bucaramanga, Colombia, dengue haemorragic fever (DHF) has become endemo-epidemic since 1992. A cross-sectional study covering a period of 10 years (February, 1992 to February, 2002) was undertaken in children under 13 years of age hospitalized at the University Hospital. Observations were recorded on the clinical features, laboratory tests and the natural development of the disease. A total of 763 patients were examined, of whom 617 were classified as having DHF according to the WHO criteria (9.1\% Grade I, 61.5\% Grade II, $21.7 \%$ Grade III and 7.5\% Grade IV). One hundred forty six patients could not be classified. The highest incidence took place in 1997, 1998 and 2001. Seventy four per cent of patients came from the metropolitan area of Bucaramanga; $48 \%$ were males; $0.3 \%$, newborns; $11.8 \%$, infants; $23 \%$, pre-school children, and $64.9 \%$, school children. The most important clinical features were fever and haemorrhagic manifestations (100\%); vomiting (60\%); abdominal pain (57\%); headache (50\%); osteomyalgia (40,8\%); hepatomegaly (33\%), and macular rash $(29 \%)$. Among the haemorragic manifestations we found petechiae (56\%); positive tourniquet test $(35 \%)$; gastrointestinal bleeding (34\%), and epistaxis (32\%). Serous effusion was found in $17.7 \%$ of cases. Alarm signs of shock were found in $29 \%$. Fifty two per cent had leucopenia and $37.3 \%$ atypic lymphocites. Among other unusual manifestations were hepatitis, encephalopathy, alithiasic cholecystitis, acute renal failure, haemophagocytic syndrome and coinfections. Of the 617 cases, 12 died $(1.5 \%)$.

Key words: dengue haemorrhagic fever, children. 
Antes de la década de 1950, la fiebre dengue no causaba gran preocupación como causa de mortalidad. En 1954, se describió la fiebre hemorrágica filipina en Manila; después, en 1956, otra epidemia similar se relacionó con el virus del dengue y se describió como una forma de la enfermedad aparentemente nueva, denominada fiebre hemorrágica por dengue (FHD) o dengue hemorrágico (1-3). Posteriormente, se llegó a la conclusión de que la enfermedad no era nueva y que desde 1780, esporádicamente se habían informado pacientes con una enfermedad clínicamente compatible con FHD (4). La enfermedad continuó propagándose en forma epidémica en el sudeste de Asia e islas del Pacífico y se volvió endémica en algunos de ellos $(4,5)$. La primera y más importante epidemia de dengue hemorrágico en América ocurrió en Cuba en 1981 (6), lo cual despertó la preocupación en la región (7). Posteriormente, casi todos los países del Caribe comenzaron a informar brotes de la enfermedad y estos casos insinuaron claramente que la FHD era una enfermedad emergente en las Américas (4,8-13). Venezuela presentó en 1989-1990 la segunda epidemia grave en el continente (14). En Colombia, el dengue estuvo ausente durante 20 años pero reapareció en 1971 (15) y en 1989 se presentaron los primeros casos de dengue hemorrágico; desde entonces han venido aumentando progresivamente en gran parte del territorio nacional $(10,16,17)$. En el departamento de Santander fue descrito el primer brote de FHD en Barrancabermeja en 1991 (18) y en 1992 describimos la epidemia inicial en niños en Bucaramanga (19), momento desde el cual ha continuado presentándose en forma endemoepidémica. El propósito del presente estudio es mostrar nuestras propias observaciones sobre las características clínicas, de laboratorio y la evolución de los niños admitidos en nuestro hospital con diagnóstico de FHD y, de esta

\footnotetext{
Correspondencia:

Gerardo González, Departamento de Pediatría, Universidad Industrial de Santander, Bucaramanga, Colombia. Teléfono: (577) 638 9404; fax: (577) 6381162 gergon@uis.edu.co
}

Recibido: 20/11/02; aceptado: 23/05/03 manera, contribuir con el conocimiento de una enfermedad que amenaza crecientemente a nuestra población.

\section{Materiales y métodos}

Este estudio descriptivo y observacional se realizó en el Hospital Universitario Ramón González Valencia (HURGV) de Bucaramanga, entidad del Estado, el cual es el centro de cuidado terciario más grande en el departamento de Santander. Bucaramanga es una ciudad situada en la Cordillera Oriental, en el nororiente de Colombia, a una altitud $1.000 \mathrm{msnm}$, clima tropical con temperatura promedio de $22^{\circ} \mathrm{C}$ y aproximadamente $1^{\prime} 000.000$ de habitantes en su área metropolitana (cuatro municipios circunvecinos).

Desde febrero de 1992, cuando se describieron los primeros casos, iniciamos la observación de los pacientes y se diseñó un formulario con variables sociodemográficas (edad, grupo de edad, género y procedencia), fechas de hospitalización y estancia hospitalaria, manifestaciones clínicas, signos hemorrágicos, derrames serosos, exámenes de laboratorio (hemograma, plaquetas y otros), confirmación diagnóstica (serología), complicaciones, tratamiento y evolución. Los grupos de edad se clasificaron en cuatro categorías: recién nacidos (menores de 1 mes), lactantes (mayores de 1 mes y menores de 2 años), preescolares (mayores de 2 años y menores de 6 años) y escolares (mayores de 6 años y menores de 13 años).

La clasificación clínica, epidemiológica e inmunológica se hizo de acuerdo con la definición de caso de la Organización Mundial de la Salud (OMS) (20), según la cual en FHD deben encontrarse todos los signos siguientes: 1) fiebre o antecedentes cercanos de fiebre aguda; 2) manifestaciones hemorrágicas que incluyan, por lo menos, una de las siguientes: prueba del torniquete positiva, petequias, equimosis o púrpura, hemorragias de las mucosas, del tracto gastrointestinal o sangrado en los sitios de punciones; 3 ) trombocitopenia $\left(100.000 / \mathrm{mm}^{3} \mathrm{o}\right.$ menos); 4) extravasación del plasma debida al aumento de la permeabilidad capilar que se manifiesta, al menos, por uno de los siguientes signos: hematocrito inicial $20 \%$ mayor que el 
correspondiente a la edad, género y población; descenso de $20 \%$ del hematocrito después del tratamiento; signos asociados con la extravasación de plasma como derrame pleural, ascitis e hipoproteinemia. El síndrome de choque por dengue incluye los cuatro criterios de dengue hemorrágico, más evidencia de colapso circulatorio que se manifiesta por los siguientes signos: pulso rápido y débil, tensión diferencial disminuida ( $20 \mathrm{~mm} \mathrm{Hg}$ o menos) o hipotensión en relación con la edad, piel fría y húmeda, y alteración del estado mental.

La clasificación por grados de gravedad de FHD se hizo también según lo recomendado por la OMS (21): grado I, prueba del torniquete positiva, como única manifestación hemorrágica; grado II, hemorragias espontáneas; grado III, falla circulatoria manifestada por pulso rápido o débil, hipotensión o tensión diferencial estrecha, piel húmeda y fría, inquietud o agitación, y grado IV, choque profundo con presión sanguínea y pulso indetectables.

La confirmación inmunológica en 1992 se hizo con la prueba de inhibición de la hemoaglutinación $(\mathrm{IH})$ (21); se consideró prueba positiva cuando el título en suero tomado antes del día 14 era mayor de 1:1.280 o cuando se evidenció un aumento de cuatro veces en las muestras pareadas; estas pruebas fueron procesadas en el Instituto Nacional de Salud (Bogotá, Colombia). A partir de 1993, se determinó por inmunoensayo enzimático de captación de anticuerpos IgM mediante la prueba de MAC-ELISA (22) realizada en el Laboratorio Departamental de la Secretaría de Salud de Santander e informada a la Unidad de Epidemiología del HURGV. Los casos no confirmados serológicamente se incluyeron de acuerdo con el criterio epidemiológico, según el cual la presencia en el mismo lugar y al mismo tiempo de otros casos confirmados de dengue se consideran probables (23).

Se incluyeron en el estudio pacientes menores de 13 años admitidos en el Departamento de Pediatría del HURGV y que egresaron con diagnóstico de dengue hemorrágico durante un período de 10 años comprendido entre febrero de 1992 y febrero de 2002. La observación de todos los pacientes se realizó directamente por uno de los autores o sus colaboradores inmediatos. El formulario se diligenció manualmente y, luego, se digitó en una base de datos diseñada en Epi-Info (versión 6.04b). Se realizó análisis univariado y de las correlaciones calculando porcentajes y proporciones de las diferentes variables estudiadas.

\section{Resultados}

\section{Clasificación}

En este periodo de 10 años estudiamos 763 pacientes, de los cuales 659 tuvieron confirmación inmunológica (639 con títulos positivos de lgM y 20 por títulos de $\mathrm{IH}$ ), los restantes 104 se consideraron probables por nexo epidemiológico. Del total de pacientes, 617 se clasificaron como FHD según los criterios de la OMS (20); 146 no se pudieron clasificar, 28 porque la trombocitopenia fue mayor de $100.000 / \mathrm{mm}^{3}$, pero tuvieron manifestaciones hemorrágicas y algún signo de fuga vascular; en los otros 118 pacientes no se pudo demostrar el criterio de fuga vascular requerido por la clasificación para FHD, pero cumplían los demás criterios y en algunos de ellos se presentaron manifestaciones inusuales, además de que tuvieron confirmación inmunológica. Según la gravedad, clasificamos 57 pacientes como grado I ( $9,2 \%)$; 380 , como grado II $(61,5 \%) ; 134$, como grado III $(21,7 \%)$, y 46 , como grado IV $(7,5 \%)$.

\section{Aspectos sociodemográficos}

En la incidencia por años se presentaron picos epidémicos en 1992, 1997, 1998 y 2001 (cuadro 1). La incidencia por meses la representamos en una curva (figura 1), en la que se destaca la mayor frecuencia de casos durante los meses de mayo a julio.

El promedio de estancia hospitalaria fue de $4,8 \mathrm{y}$ la mediana de 4 días. La procedencia de los pacientes fue la siguiente: $571(74,9 \%)$ del área metropolitana de Bucaramanga, $139(18,2 \%)$ de otros municipios de Santander y $53(6,9 \%)$ de otros departamentos. El 91,5\% vivía en el área urbana. 367 (48\%) fueron de género masculino y 396 (52\%) de género femenino. La relación hombre:mujer fue de 1:1,08. La gravedad de la FHD fue similar en ambos géneros. 
Cuadro 1. Incidencia de FHD en el niños (HURGV), Santander y Colombia, 1992-2001.

\begin{tabular}{lccc}
\hline Año & $\begin{array}{c}\text { Niños } \\
\text { (HURVG) }\end{array}$ & Santander & Colombia \\
\hline 1992 & 67 & 242 & 494 \\
1993 & 15 & 30 & 302 \\
1994 & 35 & 35 & 568 \\
1995 & 53 & 45 & 1.028 \\
1996 & 42 & 51 & 1.757 \\
1997 & 146 & 968 & 3.950 \\
1998 & 157 & 1.007 & 5.173 \\
1999 & 29 & 350 & 1.093 \\
2000 & 49 & 494 & 1.824 \\
2001 & 138 & 763 & 6.540 \\
\hline
\end{tabular}

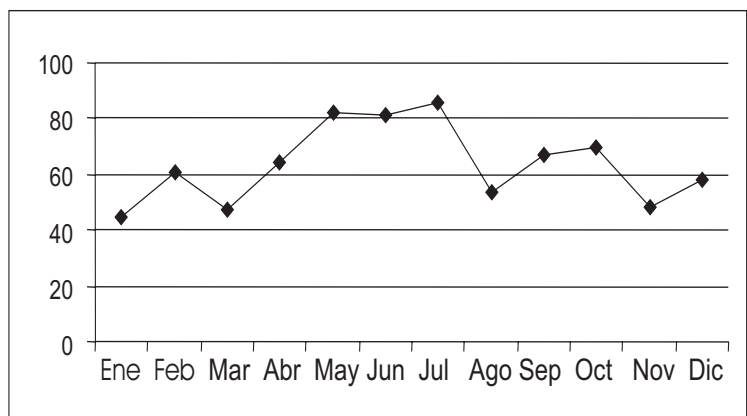

Figura 1. Frecuencia por meses en niños con FHD.

La figura 2 muestra la distribución según la edad de los pacientes incluidos en el estudio. El rango de edad fue de 0 a 13 años, con un promedio de 6,96 y una mediana de 7 . La distribución por grupos de edad fue la siguiente: recién nacidos, $2(0,3 \%)$; lactantes, 90 (11,8\%); preescolares, $176(23,1 \%)$, y escolares, $495(64,9 \%)$. La gravedad de la FHD guardó la misma proporción a la frecuencia por cada grupo de edad.

\section{Hallazgos clínicos}

Las principales manifestaciones clínicas se presentan en orden de frecuencia (cuadro 2). Las manifestaciones hemorrágicas se encontraron así: petequias $(56 \%)$, epistaxis $(32 \%)$, hematemesis $(21 \%)$, melenas $(11 \%)$, equimosis $(10 \%)$, gingivorragia ( $7 \%$ ) y hematuria (3\%); la prueba de torniquete fue positiva en $35 \%$ pero no se realizó en todos los pacientes que presentaron hemorragias espontáneas.

Se presentaron derrames serosos en 135 pacientes $(17,7 \%)$, de los cuales 80 tuvieron hidrotórax; 72, ascitis; 8, derrame pericárdico, y 5 , hidrocele. Treinta pacientes presentaron más de un derrame. En 121 pacientes (15,8\%) se observaron edemas periféricos.

Se describieron signos de alarma premonitorios de choque en 222 pacientes (29\%), algunos con más de un signo. La frecuencia de éstos fue la siguiente: 200 con dolor abdominal intenso, 43 con vómito frecuente, 38 con hipotermia, 31 con somnolencia y 20 con signos de abdomen agudo.

\section{Hallazgos paraclínicos}

Los principales hallazgos de laboratorio se describen a continuación. La distribución del recuento plaquetario se hizo agrupando los resultados así: $<20.000 / \mathrm{mm}^{3}, 135(17,7 \%) ; 20.000-$

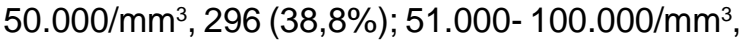

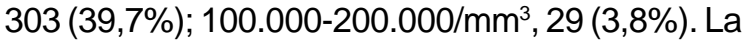

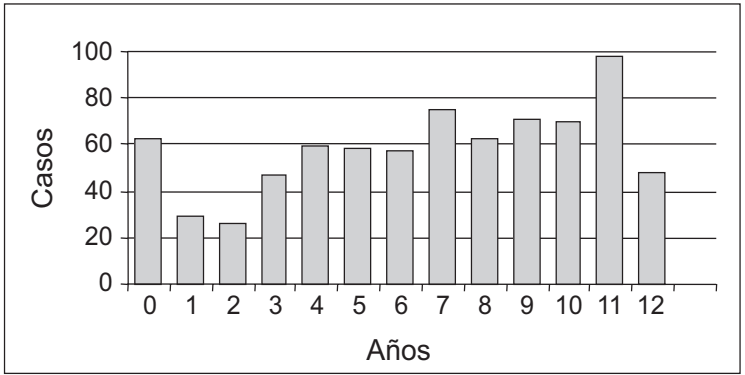

Figura 2. Distribución por edad en niños con FHD.

Cuadro 2. Manifestaciones clínicas en niños con FHD.

\begin{tabular}{lrl}
\hline Manifestación & $\mathbf{n}$ & $\%$ \\
\hline Fiebre & 763 & 100 \\
Hemorragias & 763 & 100 \\
Vómito & 458 & 60 \\
Dolor abdominal & 436 & 57 \\
Cefalea & 387 & 50,1 \\
Dolor osteomuscular & 312 & 40,8 \\
Hepatomegalia & 252 & 33 \\
Primer exantema & 183 & 24 \\
Diarrea & 122 & 16 \\
Dolor ocular & 111 & 14,5 \\
Trastornos de conciencia & 60 & 7,8 \\
Convulsiones & 56 & 7,3 \\
Náuseas & 47 & 6,1 \\
Segundo exantema & 41 & 5,4 \\
Trastornos de conducta & 23 & 3 \\
Ictericia & 6 & 0,8 \\
Esplenomegalia & 4 & 0,5 \\
\hline
\end{tabular}


menor cifra de plaquetas fue de $1.500 / \mathrm{mm}^{3}$. Establecimos correlación entre trombocitopenia y hematemesis y se encontró que en el grupo de $<20.000$ plaquetas, se presentaron $42 / 135$ (31\%) y en el grupo de 20.000-50.000 hubo 70/296 (23,6 $\%$ ), pero en el grupo $>50.000$ no se presentó hemorragia digestiva. Se demostró hemoconcentración por cifras mayores de $20 \%$ en relación con el hematocrito basal previo o posterior en 359 pacientes y por hematocrito mayor de 45 en 153. Establecimos la relación hematocrito/ hemoglobina en los pacientes a quienes se les realizaron, por lo menos, dos exámenes, encontrando que sólo 5 pacientes en el primer examen, 6 en el segundo, 5 en el tercero y 3 en el cuarto tuvieron una relación de 3,5 o mayor. En una proporción no cuantificada encontramos coincidencia entre las cifras mínimas de plaquetas y las máximas de hematocrito, principalmente en los grados III y IV. Se observó leucopenia $(<5.000$ leucocitos $/ \mathrm{mm}^{3}$ ) en $52 \%$. Leucocitosis sólo se observó en $19 \%$ de los pacientes, el resto de los recuentos leucocitarios se consideraron como cifras normales para la edad. El rango de leucocitos estuvo entre 1.700 y 35.600 . En 37,3\% se encontraron linfocitos atípicos.

Se practicaron pruebas de coagulación, tiempo de protrombina (TP) y tiempo parcial de tromboplastina (TPT) a 200 pacientes, de los cuales en 82 se encontraron prolongadas. Se analizaron las aminotransferasas en 55 pacientes; se encontraron AST elevadas en 38 (rango de 145 a $1.700 \mathrm{U} / \mathrm{L}$, con promedio de $375 \mathrm{U} / \mathrm{L}$ ) y ALT en 31 (rango de 115 a1.200 U/L, con promedio de $336 \mathrm{U} / \mathrm{L}$ ). Se determinaron las bilirrubinas en 20 pacientes, las cuales estuvieron altas a expensas de la directa en 6 casos.

Se realizó examen general de orina en 336 pacientes: normal en 288 , hematuria microscópica en 35 y proteinuria leve en 13. La creatinina se estudió en 50 pacientes (en 40 fue normal y en 10 elevada) y BUN en 43 ( 18 normal y 15 aumentada). Se practicaron otros exámenes para diagnóstico diferencial: gota gruesa, 13 (todos negativos para paludismo); antígenos febriles, 10 (negativos); se hizo mielograma en 12 pacientes, de los cuales, 5 fueron considerados por hematología como síndrome hemofagocítico. Se practicaron pruebas para hepatitis A, B y C en 5 pacientes, las cuales fueron negativas, y cultivos para descartar otras infecciones, así: hemocultivos en 17 pacientes, de los cuales 13 fueron negativos y 4 positivos; se reportaron 2 urocultivos positivos y los 3 mielocultivos tomados fueron negativos.

\section{Observaciones terapéuticas}

El manejo de todos los pacientes se hizo de acuerdo con las recomendaciones de la OMS. Se administraron líquidos endovenosos, principalmente cristaloides, a 697 pacientes; se aplicaron expansores plasmáticos a 16 pacientes; albúmina a 4; transfusiones de hemoderivados a 60 pacientes (glóbulos rojos a 29, sangre fresca a 4 , plasma a 16 , crioprecipitado a 7 y plaquetas a 10 pacientes). Recibieron antibioticoterapia 58 pacientes. Se administraron otras medicaciones, así: furosemida, 14; manejo anticonvulsivante, 33 pacientes. Se practicaron procedimientos invasores a 4 pacientes: dos toracostomías con tubo a tórax y dos laparotomías exploradoras. Veinte pacientes complicados fueron manejados en la Unidad de Cuidado Intensivo Pediátrico, 3 requirieron ventilación mecánica y 8 recibieron inotrópicos.

\section{Manifestaciones inusuales}

Doce pacientes tuvieron otras complicaciones hematológicas, de los cuales en 7 se diagnosticó coagulación intravascular diseminada y en 5 pacientes se encontró síndrome hemofagocítico; 38 cursaron con hepatitis, pero sólo en 6 se observó ictericia. Un paciente presentó insuficiencia hepática aguda. Además, 12 pacientes presentaron colecistitis acalculosa. En un paciente se demostró pancreatitis aguda; 20 pacientes presentaron signos de abdomen agudo.

Entre las manifestaciones neurológicas se observaron, en orden de frecuencia: trastornos de conciencia, convulsiones y trastornos de conducta. La frecuencia de los trastornos de conciencia fue: desorientación, 25; somnolencia, 25; estupor, 9; coma, 1. Entre los pacientes que presentaron convulsiones, 6 tenían antecedentes de crisis convulsivas (4 febriles y 2 de epilepsia en tratamiento). La presentación de los trastornos de conducta, con su respectiva frecuencia, fue: 
irritabilidad, 12; alucinaciones, 2; delirio, 1; agresividad, 2; depresión, 2; terrores, 2, y síndrome conversivo, 2 . Seis pacientes presentaron signos de encefalitis (2 con hemiparesia) y 2 meningoencefalitis. Entre los pacientes que presentaron encefalopatía secundaria a problemas metabólicos, 6 se debieron a insuficiencia renal aguda, 1 a insuficiencia hepática y 6 a trastornos hidroelectrolíticos o hipoperfusión en pacientes con choque.

Las manifestaciones neurológicas fueron más frecuentes en los lactantes, seguidos por preescolares y escolares. A 32 de estos pacientes se les practicó examen de LCR, de los cuales 31 fueron normales y sólo uno presentó pleocitosis a expensas de linfocitos. Se realizó TAC cerebral en 18 pacientes, de los cuales, 11 fueron normales y 7 con edema cerebral. Se practicó resonancia magnética a un paciente con encefalitis aguda.

Trece pacientes cursaron con edema pulmonar y 1 con síndrome de dificultad respiratoria. Diez pacientes presentaron insuficiencia renal aguda, 3 necesitaron diálisis y 1 correspondió a síndrome hemolítico urémico.

Además de los 8 pacientes con derrame pericárdico descritos entre los derrames serosos, se presentó compromiso cardiaco en 5 pacientes: 2 con miocarditis y 3 con bradicardia sinusal.

Se presentaron infecciones nosocomiales sobreagregadas al final de la fase afebril o al inicio de la convalescencia, consistentes en 10 neumonías, 10 sepsis y 2 flebitis. Se observaron las siguientes infecciones concomitantes: 14 de piel y tejidos blandos; 10 infecciones respiratorias ( 6 altas y 4 neumonías); 2 infecciones urinarias y un paciente de cada una de las siguientes: sepsis, enfermedad diarreica bacteriana, mucositis, gingivoestomatitis, vulvovaginitis y varicela reciente.

Otras enfermedades concomitantes, no infecciosas, fueron: cardiacas, 6 (5 cardiopatias congénitas, 1 bloqueo A-V); oncológicas, 2 (2 leucemias linfoides agudas que estaban en tratamiento); hematológicas, 2 (anemia de células falciformes y un paciente con tromboastenia); reumatológicas, 2 (ambas artritis reumatoidea juvenil); enfermedades respiratorias, 3 (2 con asma y una enfermedad adenomatosa quística); sólo dos pacientes se consideraron desnutridos graves.

\section{Evolución}

La gran mayoría de los pacientes evolucionaron hacia la mejoría, excepto 12 (1,5\%) que fallecieron, todos en choque profundo (grado IV). En estos pacientes no hubo diferencia en cuanto a género: 6 masculinos y 6 femeninos. Cinco de los pacientes fallecidos fueron menores de un año de edad.

\section{Discusión}

Desde que aparecieron los primeros casos de dengue hemorrágico en Bucaramanga, se inició este estudio, cuyos resultados son el producto de un seguimiento y observación durante diez años de todos los pacientes hospitalizados con esta enfermedad en el Departamento de Pediatría del hospital universitario. Bucaramanga y sus municipios vecinos conforman el área metropolitana y son considerados actualmente zona hiperendémica del dengue (24).

Para referencia de nuestro estudio, reunimos en el cuadro 1 a nuestros pacientes junto a los casos de FHD confirmados oficialmente en Colombia y el departamento de Santander, considerado el de mayor prevalencia $(25,26)$, los cuales muestran un subregistro oficial importante, como en 1995 cuando la cifra oficial fue inferior a nuestros casos confirmados. En cuanto a la incidencia, se destaca la primera epidemia que tuvimos en 1992 (19) y que despertó el interés de nuestra comunidad médica sobre el problema. Posteriormente, la enfermedad continuó presentándose en forma endémica y volvió a tener picos epidémicos en 1997, 1998 y 2001 (24-26).

Desde la aparición del primer caso de FHD en Colombia, en diciembre de 1989 , se ha observado en el país una tendencia al rápido incremento en el número de casos, pasando de 1,4 casos por 100.000 habitantes en 1994 a 5,17 casos por 100.000 en 1998, disminuyendo en 1999 y 2000 pero volviéndose a presentar una tasa similar en 2001. Entre tanto, Santander siempre ha tenido la mayor incidencia, llegando en 2001 a una tasa 
para dengue hemorrágico de 143,7 casos por 100.000 habitantes, aportados principalmente por los municipios de Bucaramanga y su área metropolitana (25). En el comienzo de 2002, el comportamiento también fue epidémico (26).

En la figura 1 mostramos la frecuencia por meses calendario, encontrando una mayor proporción durante mayo, junio y julio, los cuales corresponden en nuestro territorio a una de las épocas de lluvias. Esta misma relación se ha observado en los períodos epidémicos en el sudeste asiático $(27,28)$, lo que ha sido considerado como factor de riesgo (29) y en los pronósticos de salud pública debe tenerse en cuenta $(30,31)$.

No informamos serotipos en nuestro estudio porque la proporción de aislamientos fue muy pequeña, ya que las muestras de suero enviadas, generalmente se habían tomado después del período virémico. En relación con los serotipos circulantes durante estos años, en nuestra región se había informado que habían circulado Den-1 y Den-2 hasta 1999 (15,16); en 2000 y 2001 se aislaron Den-2 y Den-4, y a partir de agosto de 2001 también se ha aislado Den-3 en el área metropolitana de Bucaramanga $(25,32)$.

En relación con la clasificación de nuestros 763 pacientes, incluimos 617 casos que cumplían todos los criterios de la OMS (20) para clasificarlos como FHD. En éstos, el criterio de fuga vascular estuvo dado por hemoconcentración con hematocrito elevado en $20 \%$ o más en 378 pacientes; hematocrito $>45 \%$ en 153 ; derrames serosos, 135, e hipoproteinemia en 45 , aunque en muchos de los pacientes coincidía más de uno de los criterios anteriores. Estos criterios también han sido tomados por otros autores para la clasificación (33-35). Ciento cuarenta y seis pacientes no pudieron ser clasificados, 28 porque en los exámenes practicados no se demostró trombocitopenia $<100.000$, pero sí hemorragias y fuga vascular; 118 pacientes, en quienes no se pudo demostrar fuga vascular, pero sí cumplían los demás criterios para FHD. En 60 de estos pacientes encontramos aumento del hematocrito en más de $10 \%$, pero menor de $20 \%$. Algunos autores han incluido a estos pacientes como fiebre dengue con manifestaciones hemorrágicas inusuales (36), pero nosotros no lo hemos hecho porque tampoco se ajustarían a la clasificación de fiebre dengue y porque hacerlo produce un falsa sensación de seguridad que no es prudente para el manejo. Además, la administración de líquidos endovenosos de hecho altera los valores del hematocrito por lo cual la hemoconcentración es el criterio más difícil de establecer en la práctica clínica $(33,35,37,38)$. A menos que el hematocrito sea muy alto, la clasificación OMS sólo se puede hacer retrospectivamente y es de interés limitado en el manejo del paciente (35). También tuvimos pacientes que se excluyeron del estudio por no haberse demostrado ninguna hemorragia a pesar de la trombocitopenia y de otras manifestaciones de fuga vascular. El problema de clasificación fue también evidente en un informe de Tailandia, en el que pacientes clasificados como FHD no tenían hemoconcentración o menos de 100.000 plaquetas (39). Además, se han informado formas graves y fatales de dengue, las cuales no se ajustan a la definición de FHD (35,40-43). Como lo han manifestado otros autores, existen múltiples dificultades para la clasificación del dengue según los actuales criterios de la OMS (38,44-46). La OMS no tiene en cuenta en su clasificación toda la gravedad del dengue porque, además de los criterios definidos, se ha insistido en que otros criterios deben incluir las manifestaciones hemorrágicas masivas, el recuento de plaquetas de menos de 50.000, las manifestaciones neurológicas y el compromiso hepático, entre otros (35).

El $91,5 \%$ de los pacientes procedía de áreas urbanas y los restantes de áreas rurales con pequeños caseríos, lo cual está de acuerdo con los hábitos urbanos de Aedes aegypti, único vector demostrado en nuestro territorio (24). El 52\% de la población estudiada correspondió al género femenino y esta misma proporción se mantuvo cuando excluimos los pacientes que no pudieron ser clasificados. El predominio en este género es similar a lo descrito en muchos estudios anteriores (47-50), aunque en Singapur predominó el género masculino, pero incluyendo adultos (44). La edad más frecuentemente afectada, como también lo han encontrado otros autores, corresponde al 
grupo de escolares (45,47-50). Con relación al género y a la edad no hubo diferencias en cuanto a la gravedad.

El hecho de que todos los pacientes estuvieron hospitalizados, generalmente a partir de cuando se presentaron los signos de FHD, hace difícil valorar los signos y síntomas iniciales. En todos encontramos historia de fiebre antes o al inicio de la hospitalización. Por la propia definición de la enfermedad, la fiebre y alguna manifestación hemorrágica se encuentran en todos los reportes $(44,51)$. Las manifestaciones clínicas que siguieron en frecuencia a la fiebre y las hemorragias fueron: vómito, dolor abdominal y cefalea, seguidas de dolor osteomuscular y hepatomegalia. La cefalea es difícil de evaluar en lactantes y preescolares (52). El primer exantema se presenta generalmente en la fase febril y puede ser pruriginoso, pero, a veces es inaparente; lo observamos sólo en $24 \%$, mientras en otros estudios se encuentra en mayor proporción; también tuvimos dos pacientes con erupción urticariforme como fue observado en China (53). El segundo exantema confluente y petequial con áreas pálidas como 'islas blancas en un mar rojo' (54), aparece al final de la fase afebril y principio de la convalecencia y se ha considerado como un signo de recuperación (55). Nosotros ya habíamos descrito este segundo exantema y creemos que podría llegar a considerarse casi patognomónico del dengue (19). $16 \%$ de nuestros pacientes, principalmente lactantes, presentaron diarrea, como lo mencionan otros estudios pero en menor proporción $(45,47)$.

Las petequias fueron el signo hemorrágico más frecuente $(56 \%)$, similar a otros estudios: $52,3 \%$ en Singapur (44) y $45,6 \%$ en Puerto Rico (56). Cuando se nos refería ausencia de hemorragias, con frecuencia (no determinada en nuestro protocolo) si se examinaba cuidadosamente se encontraron petequias especialmente en paladar, por lo cual pueden pasar desapercibidas, como lo han señalado en Cuba (45). Le siguieron en frecuencia otras manifestaciones hemorrágicas como la epistaxis (32\%) y nos llamó la atención que sólo se presentó hematemesis en los grupos con menos de 50.000 plaquetas, lo cual se ha observado en otros estudios de adultos $(46,53,57)$, mientras que la hematuria la hallamos en muy pocos de nuestros pacientes ( $3 \%)$, diferente a lo informado en Puerto Rico, donde encontraron $51,9 \%$ de hematuria microscópica (56). Se encontraron hemorragias menos frecuentes como menorragias, hemorragia subconjuntival, hematomas en sitios de venopunción y hemorragia intracraneana. Todos los pacientes con coagulación intravascular diseminada fueron FHD grado IV (choque), de los cuales 6 fueron refractarios al tratamiento y, finalmente, fallecieron.

La relación hematocrito/hemoglobina no se encontró elevada como en otros trabajos (58) y no tuvo importancia en nuestros pacientes. En cambio, lo que sí tiene importancia sobre estos parámetros es el tiempo en que concurre el valor mínimo de trombocitopenia con la elevación máxima del hematocrito porque cuando coinciden es un signo de alarma de choque inminente, lo cual pudimos corroborar en nuestro estudio y se ha afirmado anteriormente (59). No hubo correlación significativa de las cifras de trombocitopenia con la gravedad del sangrado, excepto con hematemesis, lo cual ha sido encontrado por otros autores en relación con todas las hemorragias digestivas $(44,60)$.

Fue importante la cifra de leucopenia hallada en más de la mitad de los pacientes, lo cual puede presentarse en diferentes fases y constituye un parámetro importante en el diagnóstico clínico. Los linfocitos atípicos se han encontrado con mayor frecuencia en FHD que en fiebre dengue (59).

Entre los derrames serosos, el más frecuentemente encontrado fue el hidrotórax. Cuando se presentaron edemas periféricos, se relacionaron en la mayoría de los casos con sobrecarga de líquidos, como se ha mencionado en la literatura (55). Los signos de alarma, premonitorios de choque descritos desde la epidemia de Cuba (58), fueron útiles en el manejo de nuestros pacientes, especialmente el dolor abdominal intenso, que fue el más frecuente.

Se observaron diferentes manifestaciones inusuales en nuestro estudio. Entre las hematológicas, encontramos 5 pacientes con síndrome hemofagocítico, quienes después de 
estabilizarse hemodinámicamente evolucionaron en forma atípica con fiebre prolongada y citopenias periféricas que requirieron aspirado de médula ósea para el diagnóstico diferencial con otras entidades; se encontró hemofagocitosis, la cual consideramos asociada con el dengue. Este síndrome sólo se había informado anteriormente en 2 casos $(61,62)$; nosotros lo hicimos en un primer informe de 3 pacientes (63).

Tuvimos 38 pacientes que interpretamos como hepatitis por dengue, en quienes se descartaron otras causas posibles y, de ellos, sólo 6 cursaron con ictericia e hiperbilirrubinemia a expensas de la bilirrubina directa. El diagnóstico se hizo con base en las aminotransferasas elevadas, principalmente AST, como se ha informado en otros estudios $(37,64)$. Un paciente con hepatitis evolucionó a insuficiencia hepática con encefalopatía. El compromiso hepático se ha asociado hasta en $50 \%$ de las formas graves (35), cada día se informan más casos (65-67) y se reafirma que la hepatomegalia con elevación moderada de aminotransferasas se considera hepatitis por dengue, generalmente anictérica y es una entidad autolimitada (65).

Los 12 pacientes con diagnóstico de colecistitis acalculosa se demostraron por ultrasonido (68) y a 4 pacientes con esta complicación también se les comprobó hepatitis mediante pruebas de función hepática, lo cual también se ha informado (69). Debe reiterarse que estos pacientes no necesitan intervención quirúrgica y sólo con manejo médico tienen buena evolución. Sólo un paciente del estudio presentó manifestaciones clínicas y de laboratorio de pancreatitis aguda, recibió tratamiento médico y evolucionó satisfactoriamente, similar a un paciente informado en Indonesia (70).

De los 20 pacientes con abdomen agudo, 4 fueron intervenidos con laparatomía exploradora y se encontró ascitis gelatinosa, lo cual asociado con trombocitopenia postoperatoria hizo sospechar FHD, confirmado posteriormente por IgM. Los pacientes con abdomen agudo evolucionaron satisfactoriamente con manejo médico, excepto un lactante que falleció en el postoperatorio de la laparotomía exploradora. El dolor abdominal grave en el dengue se ha atribuido a varios factores como distensión de la cápsula hepática, estimulación de plexos nerviosos por la distensión del espacio retroperitoneal a causa del edema (58) o por perfusión mesentérica disminuida (71) y debe tenerse en cuenta en el diagnóstico diferencial del abdomen agudo en zonas endémicas de dengue, para evitar laparotomías innecesarias (19).

Las manifestaciones neurológicas en infecciones por dengue pueden presentarse tanto en fiebre dengue como en FHD, en cualquier estadio, con cualquier gravedad y por cualquier serotipo (72). Nuestras observaciones clínicas son similares a lo que se está informando en todas las latitudes; las alteraciones de conciencia y convulsiones son las más comunes. Otras manifestaciones neurológicas asociadas con dengue como encefalopatía, meningismo, mononeuropatías, polineuropatías, delirio, espasticidad, parálisis y alteración de nervios craneales, síndrome de Guillain Barre, mielitis transversa, mielitis postinfecciosa y desórdenes del comportamiento han sido informadas anteriormente $(41,73)$. Debido a que el comienzo de la encefalitis aparece temprano en el curso de la enfermedad, coincidiendo con la fase virémica, se ha postulado que el virus cruza la barrera hematoencefálica, invade directamente el cerebro causando encefalitis y el compromiso cerebral puede ser generalizado o focalizado como lo sugieren los hallazgos de TAC (41). Por lo anterior, en áreas endémicas, el virus dengue debe considerarse como otra causa posible de encefalopatía $(72,73)$.

La mayoría de las complicaciones pulmonares se observaron después de la administración de líquidos endovenosos o transfusiones. Los pacientes con edema pulmonar mostraron, además, edemas periféricos, signos de hipervolemia y cursaron con presión arterial en percentiles superiores normales o elevados para su edad y fueron considerados como manifestación de hipervolemia en los casos en que se prolongó la terapia con líquidos endovenosos después de la fase de fuga vascular, lo cual ya ha sido referido anteriormente (74), pero también se ha encontrado edema pulmonar en pacientes con hidrotórax bilateral, así como en 
períodos de choque, lo cual se correlaciona con la propia fuga vascular (58) o puede presentarse por falla renal (46). Una paciente presentó al final de la fase afebril síndrome de dificultad respiratoria y requirió ventilación mecánica, lo cual, aunque es una complicación poco frecuente, ha sido descrita en niños $(75,76)$.

De nuestros pacientes con insuficiencia renal aguda, 3 requirieron diálisis peritoneal, uno se complicó con insuficiencia cardiaca y edema pulmonar y otro se manifestó como síndrome hemolítico urémico. Un paciente presentó glomerulonefritis aguda posdengue, lo cual también ha sido informado (46).

Las coinfecciones pueden dificultar el diagnóstico de dengue o hacer sospechar que se trata de dengue con manifestaciones inusuales, retardando así el manejo precoz de la infección (77). La coinfección observada en nuestro estudio con mayor frecuencia fue la infección de piel y tejidos blandos, seguida de infecciones respiratorias. Las infecciones sobreagregadas se presentaron en pacientes con FHD/choque después de la estabilización hemodinámica, la mayoría manejadas en UCIP con procedimientos invasivos o reanimación; también, en pacientes que tuvieron estancias prolongadas por manifestaciones inusuales o complicaciones del dengue. La infección nosocomial más frecuente fue la neumonía seguida de la sepsis. Todos los pacientes con coinfecciones o infecciones sobreagregadas recibieron tratamiento adecuado con antibioticoterapia. En el primer informe que menciona infecciones duales, se postula que las causas posibles en pacientes con dengue incluyen coincidencia de infecciones o predisposición por efectos inmunes del virus dengue (77).

Las enfermedades concomitantes no infecciosas pueden complicar el diagnóstico y manejo del dengue. En nuestro estudio tuvimos un niño con cardiopatía congénita cianógena diagnosticada previamente (tetralogía de Fallot), que presentó hematocrito muy elevado y requirió flebotomía. Los dos pacientes con diagnóstico previo de leucemia ingresaron con diagnóstico de sepsis y neutropenia febril severa, tuvieron cultivos negativos y la sospecha de dengue se hizo en los días 6 y 7 de la enfermedad, cuando presentaron el segundo exantema característico; se tomaron muestras para serología lgM y se confirmó posteriormente el diagnóstico de dengue. Los dos pacientes con diagnóstico de artritis reumatoidea juvenil en tratamiento con antiinflamatorios no esteroideos presentaron sangrados masivos gastrointestinales y uno de ellas falleció en coagulación intravascular diseminada. El paciente con anemia de células falciformes falleció en choque refractario. El paciente con tromboastenia presentó sangrados inusuales. Los dos pacientes desnutridos graves se complicaron con infecciones sobreagregadas consistentes en neumonía y sepsis. Un paciente escolar de 10 años con bloqueo auriculoventricular se complicó con taquicardia ventricular, choque y paro cardiaco que requirió reanimación y manejo en UCIP con tratamiento específico por cardiología.

Algunas enfermedades crónicas como diabetes mellitus, asma bronquial, anemia drepanocítica, epilepsia y cardiopatías congénitas han sido consideradas como factores de riesgo para FHD y se han informado en otros estudios $(52,58)$.

En nuestra serie fallecieron 12 pacientes, todos con FHD grado IV (en choque profundo). Seis se complicaron con coagulación intravascular diseminada, todos con hemorragia digestiva masiva y dos con hemorragia pulmonar. Uno hizo sepsis por Gram negativos, dos tuvieron enfermedades concomitantes no infecciosas: uno con artriris reumatoidea juvenil en tratamiento con antiinflamatorios no esteroideos y otro con anemia de células falciformes. Uno de los pacientes fallecidos hizo signos de abdomen agudo y le fue practicada laparotomía exploradora. La mortalidad en hospitales con amplia experiencia puede ser baja, pero puede elevarse significativamente en los pacientes con choque establecido, en caso de hemorragias masivas o con manifestaciones inusuales como compromiso cardíaco, hepático, renal o neurológico (75-79). Seis de nuestros pacientes fallecidos correspondieron al grupo de lactantes, cuatro escolares, un preescolar y un recién nacido, lo cual coincide con lo observado en Puerto Rico (37). La estancia hospitalaria en la mitad de los pacientes fallecidos fue menor de 
24 horas, 3 pacientes estuvieron menos de 3 días y 3 , más de 3 días. El paciente con mayor tiempo de hospitalización fue el neonato. Todos fueron atendidos en el servicio de urgencias, la mitad fueron trasladados a la UCIP y los demás no pudieron ser trasladados por falta de cama disponible en dicho servicio. Cinco pacientes empeoraron durante el traslado de hospitales regionales al hospital universitario y uno murió durante el traslado de este hospital a la UCIP de otra institución local. Los estudios similares han mostrado que en la mortalidad influye no solamente la gravedad sino la oportunidad del traslado y las adecuadas condiciones del mismo (36).

Se practicó autopsia a 2 pacientes (un neonato y un lactante), en quienes los principales hallazgos fueron derrames serosos de aspecto gelatinoso, microhemorragias viscerales generalizadas, evidencia morfológica de edema cerebral y, en hígado, congestión generalizada con extravasación eritrocitaria, esteatosis microvacuolar focal e hiperplasia de células de Kupffer. En 2 pacientes se practicó biopsia hepática postmortem, que mostró los mismos hallazgos anteriores.

En nuestro estudio hemos anotado que Bucaramanga y su área metropolitana es la ciudad de Colombia en la que mayor impacto ha tenido la FHD; actualmente se la considera área hiperendémica y la mayoría de sus médicos reconocen y manejan bien la enfermedad. Existe dificultad para la clasificación de acuerdo con los criterios actuales, pero esto no debe interferir con el adecuado manejo de los pacientes. Las coinfecciones e infecciones sobreagregadas pueden confundir el diagnóstico. Las manifestaciones inusuales son muy importantes en el diagnóstico y manejo del dengue hemorrágico y deberían tenerse en cuenta en los criterios de clasificación. La mortalidad aumenta con el retardo en el inicio del manejo, lo mismo que los inadecuados traslados en fase de choque.

\section{Agradecimientos}

A los médicos pediatras y residentes del Departamento de Pediatría del HURGV y la UIS, a la secretaria de Pediatría del HURGV, a la Unidad de Epidemiología del HURGV y al Laboratorio
Departamental de la Secretaría de Salud de Santander.

\section{Referencias}

1. Hammon WM, Rudnick A, Sather GE. Viruses associated with epidemic hemorrhagic fevers of the Philippines and Thailand. Science 1960;131:1102-3.

2. Halstead SB. Mosquito-borne haemorrhagic fevers of South and South-East Asia. Bull World Health Organ 1966;35:3-15.

3. Hammon WM. Dengue hemorrhagic fever - do we know its cause? Am J Trop Med Hyg 1973;22:82-91.

4. Gubler DJ. Dengue and dengue hemorrhagic fever: its history and resurgence as a global public problem. En: Gubler DJ, Kuno G, editors. Dengue and dengue hemorrhagic fever. Wallingford, UK: CAB International; 1997. p.1-22.

5. Halstead SB. Epidemiology of dengue and dengue hemorrhagic fever. En: Gubler DJ, Kuno G, editors. Dengue and dengue hemorrhagic fever. Wallingford, UK: CAB International; 1997. p.23-44.

6. Kouri G, Guzmán MG, Bravo J. Hemorrhagic dengue in Cuba: history of an epidemic. Bull Pan Am Health Organ 1986;20:24-30.

7. Kouri GP, Guzmán MG, Bravo JR, Triana C. Dengue haemorrhagic fever/dengue shock syndrome: lessons from the Cuban epidemic, 1981. Bull World Health Organ 1989;67:375-80.

8. Pinheiro FP. Dengue in the Americas, 1980-1987. Epidemiol Bull 1989;10:1-8.

9. Organización Panamericana de la Salud. El dengue y la fiebre hemorrágica de dengue en las Americas: una visión general del problema. Bol Epidemiol OPS 1992;13:9-10.

10. Organización Panamericana de la Salud. Dengue y fiebre hemorrágica del dengue, 1996. Bol Epidemiol OPS 1996;17:12-4.

11. Organización Panamericana de la Salud. Resurgimiento del dengue en las Américas. Bol Epidemiol OPS 1997;18:1-6.

12. Organización Panamericana de la Salud. El dengue en Centroamérica: las epidemias de 2000. Bol Epidemiol OPS 2000;21:4-8.

13. Isturiz RE, Gubler DJ, Brea del Castillo J. Dengue and dengue hemorrhagic fever in Latin America and the Caribbean. Infect Dis Clin North Am 2000;14:12-140.

14. Communicable Diseases Program, PAHO. Dengue hemorrhagic fever in Venezuela. Epidemiol Bull (PAHO) 1990;11:7-9.

15. Boshell J, Groot H, Gacharná M, Márquez G, González M, Gaitán M, et al. Dengue en Colombia. Biomédica (Bogotá) 1986;6:101-6. 
16. Jaramillo C, Alvarez G, Granados R. Dengue y dengue hemorrágico en Colombia desde la colonia hasta 1995. Tribuna Médica (Bogotá) 1997;95:45-54.

17. Ministerio de Salud, Instituto Nacional de Salud. Evaluación epidemiológica del dengue hemorrágico en Colombia. Inf Quinc Epidemiol Nac 1997;2:274-6.

18. Villar LA, Machado CA, Páez J, Renjifo AM. Fiebre hemorrágica del dengue (FHD). Descripción de un brote en Barrancabermeja (Santander). Memorias, I Congreso de Infectología; mayo de 1992; Bogotá, Colombia; p.42.

19. González G, Méndez A. Dengue hemorrágico en niños. Estudio de la epidemia de 1992 en Bucaramanga. Pediatría (Colombia) 1994;29:33-41.

20. World Health Organization. Clinical diagnosis. En: WHO. Dengue haemorrhagic fever: diagnosis, treatment, prevention and control. $2^{\text {nd }}$ edition. Geneva: WHO; 1997. p.12-23.

21. Clarke DH, Casals J. Techniques for hemagglutination and hemagglutination-inhibition with arthropod-borne viruses. Am J Trop Med Hyg 1958;7:561-73.

22. Kuno G, Gubler DJ. Detecting artificial anti-dengue IgM immune complexes using an enzyme-linked immunosorbent assay. Am J Trop Med Hyg 1987;36: 153-9.

23. Pan American Health Organization. Case definitions. Dengue fever. Epidemiol Bull 2000;21:14-5.

24. Ministerio de Salud, Colombia. Comportamiento por regiones del dengue en el 2001. Sivigila, Boletín Epidemiológico Semanal, 2002; № 2. Disponible en: URL: http://www.col.ops-oms.org/sivigila/2002/ BOLE02_02.htm.

25. Ministerio de Salud, Colombia. Prevención y control del dengue. Sivigila, Boletín Epidemiológico Semanal, 2001; № 42. Disponible en: URL: http://www.col.opsoms.org/sivigila/2001/BOLE42_2001.htm.

26. Ministerio de Salud, Colombia. Eventos de notificación: tendencia del primer trimestre del 2002. Sivigila, Boletín Epidemiológico Semanal, 2002; № 27. Disponible en: URL: http://www.col.ops-oms.org/sivigila/ 2002/BOLE27_02.htm.

27. Corwin AL, Larasati RP, Bangs MJ, Wuryadi S, Arjoso S, Sukri N et al. Epidemic dengue transmission in southern Sumatra, Indonesia. Trans R Soc Trop Med Hyg 2001;95:257-65.

28. Li CF, Lim TW, Han LL, Fang R. Rainfall, abundance of Aedes aegypti and dengue infection in Selangor, Malaysia. Southeast Asian J Trop Med Public Health 1985; $16: 560-8$

29. Koopman JS, Prevots DR, Vaca Marín MA, Gómez Dantes $H$, Zárate Aquino ML, Longini IM Jr, Sepúlveda Amor J. Determinants and predictors of dengue infection in Mexico. Am J Epidemiol 1991;133: 1168-78.

30. Githeko AK, Lindsay SW, Confalonieri UE, Patz JA. Climate change and vector-borne diseases: a regional analysis. Bull World Health Org 2000;78:1136-47.

31. Jetten TH, Focks DA. Potential changes in the distribution of dengue transmission under climate warming. Am J Trop Med Hyg 1997;57:285-97.

32. Ocazionez R, Alvarez A, Cortés F, Fábregas R, Acosta L. Asociación entre circulación de serotipos de virus dengue y ocurrencia de epidemias con aumento de la severidad. Infectio 2002;6:110.

33. Zagne SM, Alves VG, Nogueira RM, Miagostovich MP, Lampe E, Tavares W. Dengue haemorrhagic fever in the state of Rio de Janeiro, Brazil: a study of 56 confirmed cases. Trans R Soc Trop Med Hyg 1994;88: 677-9.

34. Deparis X, Murgue B, Roche C, Cassar O, Chungue E. Changing clinical and biological manifestations of dengue during the dengue-2 epidemic in French Polynesia in 1996/97 - description and analysis in a prospective study. Trop Med Int Health 1998;3:85965.

35. Murgue B, Deparis X, Chungue E, Cassar O, Roche C. Dengue: an evaluation of dengue severity in French Polynesia based on an analysis of 403 laboratoryconfirmed cases. Trop Med Int Health 1999;4:765-73.

36. Kabra SK, Jain Y, Pandey RM, Madhulika, Singhal T, Tripathi P, Broor S, Seth P, Seth V. Dengue haemorrhagic fever in children in the 1996 Delhi epidemic. Trans R Soc Trop Med Hyg 1999;93:294-8.

37. Dietz V, Gubler DJ, Ortiz S, Kuno G, Casta-Vélez A, Sather GE, G;omez I, Vergne E. The 1986 dengue and dengue hemorrhagic fever epidemic in Puerto Rico: epidemiologic and clinical observations. PR Health Sci J 1996;15:201-10.

38. Rigau-Pérez JG. Case definition for dengue hemorrhagic fever. Pediatr Infect Dis J 1999;18:80.

39. Kalayanarooj S, Vaughn DW, Nimmannitya S, Green $\mathrm{S}$, Suntayakorn $\mathrm{S}$, Kunentrasai $\mathbf{N}$ et al. Early clinical and laboratory indicators of acute dengue illness. J Infect Dis 1997;176:313-21.

40. George R, Liam CK, Chua CT, Lam SK, Pang T, Geethan R, Foo LS. Unusual clinical manifestations of dengue virus infection. Southeast Asian J Trop Med Public Health 1988;19:585-90.

41. Lum LC, Lam SK, Choy YS, George R, Harun F. Dengue encephalitis: a true entity? Am J Trop Med Hyg 1996:54:256-9.

42. Chimelli L, Hahn MD, Netto MB, Ramos RG, Dias M, Gray F. Dengue: neuropathological findings in 5 fatal cases from Brazil. Clin Neuropathol 1990;9:157-62. 
43. Hommel D, Talarmin A, Deubel V, Reynes JM, Drouet MT, Sarthou JL, Hulin A. Dengue encephalitis in French Guiana. Res Virol 1998;149:235-8.

44. Tai DY, Chee YC, Chan KW. The natural history of dengue illness based on a study of hospitalised patients in Singapore. Singapore Med J 1999;40:238-42.

45. Rodríguez O, Pérez A, Despaigne A, Irarragirri CA. Caracterización de pacientes con diagnóstico presuntivo de dengue en el brote epidémico del año 1997. Rev Cubana Med Trop 2001;53:24-7.

46. George R, Lum LC. Clinical spectrum of dengue infection. En: Gubler DJ, Kuno G, editors. Dengue and dengue hemorrhagic fever. Wallingford, UK: $C A B$ International; 1997. p.89-113.

47. Vargas ME, Aguirre TM, Palacios H. Características clínicas de la fiebre de dengue en niños durante el brote epidémico en Santiago de Cuba. Rev Cubana Med Trop 2001;53:20-3.

48. Halstead. Fiebre por dengue hemorrágico: un problema de la salud pública y un campo de investigación. Bol OMS 1980;58:1-21.

49. Moraes L, Baeta S, Costa M. Encuesta serológica sobre el dengue entre escolares de Río de Janeiro, Brasil, 1986 y 1987. Bol Of Sanit Panam 1991;111:52532.

50. Kourí G, Valdés M, Argüello L, Guzmán MG, Valdés L, Soler M, et al. Epidemia de dengue en Nicaragua, 1985. Rev Inst Med Trop Sao Paulo 1991;33:365-71.

51. Chin CK, Kang BH, Liew BK, Cheah PC, Nair R, Lam SK. Protocol for out-patient management of dengue illness in young adults. J Trop Med Hyg 1993;96:259-63

52. Palacios H, Vargas ME, Aguirre TM. Dengue hemorrágico en dengue primario. Rev Cubana Med Trop 2001;53:59-62.

53. Qiu FX, Gubler DJ, Liu JC, Chen QQ. Dengue in China: a clinical review. Bull World Health Organ 1993;71:349-59.

54. Kautner I, Robinson MJ, Kuhnle U. Dengue virus infection: epidemiology, pathogenesis, clinical presentation, diagnosis, and prevention. J Pediatr 1997; 131:516-24.

55. World Health Organization. Regional Office for SouthEast Asia. Guidelines for treatment of dengue/dengue haemorrhagic fever in small hospitals. New Delhi. WHO 1999. Disponible en: URL: http://w3.whosea.org/ techinfo/pdf/dengue.pdf.

56. Rigau-Pérez JG. Manifestaciones clínicas del dengue hemorrágico en Puerto Rico, 1990-1991. Rev Panam Salud Pública 1997;1:435-43.

57. Fagbami AH, Mataika JU, Shrestha M, Gubler DJ. Dengue type 1 epidemic with haemorrhagic manifestations in Fiji, 1989-90. Bull World Health Organ 1995;73:291-7.

58. Martínez E. Dengue hemorrágico en niños. Bogotá: Instituto Nacional de Salud; 1990. p.29-120.

59. Nimmannitya S. Dengue hemorrhagic fever: disorders of hemostasis. Disponible en: URL: http://haem.nus.edu. sg/ishapd/1999/50.pdf .

60. Tsai CJ, Kuo CH, Chen PC, Changcheng CS. Upper gastrointestinal bleeding in dengue fever. Am J Gastroenterol 1991;86:33-5.

61. Ramanathan M, Duraisamy G. Haemophagocytosis in dengue haemorrhagic fever: a case report. Ann Acad Med Singapore 1991;20:803-4.

62. Djossou F, Malvy D, Delbrel X, Germain P, Etienne G, Longy-Boursier M, et al. Haemophagocytosis in dengue fever: a case report. Proceedings, XV International Congress for Tropical Medicine and Malaria; 2000, Aug 20-26; Cartagena, Colombia; 2000. Vol 2. p.126

63. Rueda E, Méndez A, González G. Síndrome hemofagocítico asociado a dengue hemorrágico. Biomédica (Bogotá) 2002;22:160-6.

64. Gomber S, Ramachandran VG, Kumar S, Agarwal KN, Gupta P, Gupta P, Dewan DK. Hematological observations as diagnostic markers in dengue hemorrhagic fever a reappraisal. Indian Pediatr 2001;38: 477-81.

65. del Valle S, Pinera M, Guasch F. Hepatitis reactiva por virus del dengue hemorrágico. Rev Cubana Med Trop 2001;53:28-31.

66. Nguyen TL, Nguyen TH, Tieu NT. The impact of dengue haemorrhagic fever on liver function. Res Virol 1997; 148:273-7.

67. Wahid SF, Sanusi S, Zawawi MM, Ali RA. A comparison of the pattern of liver involvement in dengue hemorrhagic fever with classic dengue fever. Southeast Asian J Trop Med Public Health 2000;31:259-63.

68. Setiawan MW, Samsi TK, Wulur H, Sugianto D, Pool TN. Dengue haemorrhagic fever: ultrasound as an aid to predict the severity of the disease. Pediatr Radiol 1998;28:1-4.

69. Sood A, Midha V, Sood N, Kaushal V. Acalculous cholecystitis as an atypical presentation of dengue fever. Am J Gastroenterol 2000;95:3316-7.

70. Jusuf $H$, Sudjana $P$, Djumhana $A$, Abdurachman SA. DHF with complication of acute pancreatitis related hyperglycemia: a case report. Southeast Asian J Trop Med Public Health 1998;29:367-9.

71. Santiago A, Fernández A. Dengue in children: critical points in management. PR Health Sci J 2001;20:343-6.

72. Kankirawatana $\mathbf{P}$, Chokephaibulkit K, Puthavathana P, Yoksan S, Apintanapong S, Pong-thapisit 
V. Dengue infection presenting with central nervous system manifestation. J Child Neurol 2000;15: 544-7.

73. Solomon T, Dung NM, Vaughn DW, Kneen R, Thao LT, Raengsakulrach B, et al. Neurological manifestations of dengue infection. Lancet 2000;355: 1053-9.

74. Nimmannitya S. Dengue hemorrhagic fever: dianosis and management. En: Gubler DJ, Kuno G, editors. Dengue and dengue hemorrhagic fever. Wallingford, UK: CAB International; 1997. p.133-45.

75. Lum LC, Thong MK, Cheah YK, Lam SK. Dengueassociated adult respiratory distress syndrome. Ann Trop Paediatr 1995;15:335-9.
76. Thisyakorn U, Thisyakorn C. Dengue infection with unusual manifestations. J Med Assoc Thai 1994;77:410-3.

77. Pancharoen C, Thisyakorn U. Coinfections in dengue patients. Pediatr Infect Dis J 1998;17:81-2.

78. Rigau-Pérez JG, Clark GG, Gubler DJ, Reiter P, Sanders EJ, Vorndam AV. Dengue y fiebre hemorrágica del dengue. Lancet 1999;34:111-7.

79. Sumarmo, Wulur H, Jahja E, Gubler DJ, Suharyono W, Sorensen K. Clinical observations on virologically confirmed fatal dengue infections in Jakarta, Indonesia. Bull World Health Organ 1983;61:693-701. 